\title{
Reply to a letter to the editor referencing "Breast cancer survivors' perspectives on a home-based physical activity intervention utilizing wearable technology"
}

\author{
Renee L. Kokts-Porietis ${ }^{1,2} \cdot$ Chelsea R. Stone ${ }^{1,2} \cdot$ Christine M. Friedenreich $^{1,2,3,4} \cdot$ Alyssa Froese $^{1}$ • \\ Meghan McDonough ${ }^{4}$. Jessica McNeil ${ }^{1}$ (1)
}

Received: 22 November 2019 / Accepted: 23 December 2019 / Published online: 7 January 2020

(C) Springer-Verlag GmbH Germany, part of Springer Nature 2019

We would like to thank Kajiwara and colleagues [1] for their letter to the editor, which continues the discussion on breast cancer survivors' use of wearable technology in home-based settings to promote physical activity behavior change.

Kajiwara et al. [1] highlight the importance of assessing participant acceptance of wearable technology. In the Breast Cancer and Physical Activity Level (BC-PAL) pilot trial, acceptance or wear time of the activity tracker was not directly assessed [2]. However, the participants' perspectives of wearable technology were expressed in the semi-structured interviews, as presented by Kokts-Porietis et al. [3]. Similar to Puri et al. [4], equipment characteristics such as appearance of the device and ease of use played an important role in the participants' acceptance of the activity trackers. For instance, the pink activity trackers acted as a reminder of breast cancer for some of the participants, which reduced their interest in wearing the device [3]. Moreover, when participants felt that the results from the activity tracker did not truly reflect their physical activity participation, their acceptance and confidence in the device were also diminished [3].

We agree with Kajiwara et al. [1] that future studies should utilize wearable activity trackers to collect objective physical activity data and monitor adherence to a particular exercise

Jessica McNeil

Jessica.McNei12@albertahealthservices.ca

1 Department of Cancer Epidemiology and Prevention Research, Alberta Health Services, Holy Cross Centre, 2210 2nd SW, Calgary, Alberta T2S 3C3, Canada

2 Department of Community Health Sciences, Cumming School of Medicine, University of Calgary, Calgary, Alberta, Canada

3 Department of Oncology, Cumming School of Medicine, University of Calgary, Calgary, Alberta, Canada

4 Faculty of Kinesiology, University of Calgary, Calgary, Alberta, Canada prescription. That said, it is important to recognize that the presence of a potential feeling of "disconnect" between self and technology, as well as reduced feelings of confidence in the data provided by the activity tracker, may act as barriers for the long-term acceptance of these devices. Further understanding of features that facilitate long-term acceptance of wearable technology as an intervention tool that promotes self-awareness and reinforces physical activity behavior change is needed, especially given the increasing availability and rapid technological advancements of these devices.

\section{Compliance with ethical standards}

Conflict of interest The authors declare that they have no conflict of interest.

\section{References}

1. Kajiwara K, Kako J, Noto H, Oosono Y, Kobayashi M (2019) Reply to: Breast cancer survivors' perspectives on a home-based physical activity intervention utilizing wearable technology. Support Care Cancer. https://doi.org/10.1007/s00520-019-05125-0

2. McNeil J, Brenner DR, Stone CR, O'Reilly R, Ruan Y, Vallance JK, Courneya KS, Thorpe KE, Klein DJ, Friedenreich CM (2019) Activity tracker to prescribe various exercise intensities in breast cancer survivors. Med Sci Sports Exerc 51(5):930-940. https://doi. org/10.1249/mss.0000000000001890

3. Kokts-Porietis RL, Stone CR, Friedenreich CM, Froese A, McDonough M, McNeil J (2019) Breast cancer survivors' perspectives on a home-based physical activity intervention utilizing wearable technology. Support Care Cancer 27(8):2885-2892. https://doi. org/10.1007/s00520-018-4581-7

4. Puri A, Kim B, Nguyen O, Stolee P, Tung J, Lee J (2017) User acceptance of wrist-worn activity trackers among communitydwelling older adults: mixed method study. JMIR mHealth uHealth 5(11):e173. https://doi.org/10.2196/mhealth.8211

Publisher's note Springer Nature remains neutral with regard to jurisdictional claims in published maps and institutional affiliations. 